\title{
Control of Solar Sail Periodic orbits in the Elliptic Three-Body Problem
}

\author{
James D. Biggs * Colin R. McInnes ${ }^{\dagger}$ Thomas Waters ${ }^{\ddagger}$
}

\section{Introduction}

A Solar sail consists essentially of a large mirror, which uses the momentum change due to photons reflecting off the sail for its impulse. Solar sails are therefore unique spacecraft as they do not require fuel for propulsion. ${ }^{1}$ In this note we consider using the solar sail to continuously maintain a periodic orbit above the ecliptic plane using variations in the sail's orientation. Positioning a spacecraft continuously above the ecliptic would allow continuous observation and communication with the poles.

In Waters and McInnes ${ }^{2}$ the authors identify families of periodic orbits in the solar sail Circular Restricted Three-Body Problem (CRTBP). In this note we model the solar sail in the context of the Elliptical Restricted Three-Body problem (ERTBP) with the Earth and Sun as the two primaries and where the solar sail is the third massless body. The generalization to the solar sail ERTBP is considered in Baoyin and McInnes. ${ }^{3}$ This highlights that a study of the elliptic case is necessary, firstly because it is a more realistic model than the circular case and secondly because there are fundamental differences between the two cases. To begin with there are no equilibrium points above the ecliptic in the solar sail ERTBP. ${ }^{3}$ Additionally, the equations of motion contain the time explicitly i.e. through the true anomaly $f$ and are therefore non-autonomous. This implies that any periodic solution of the solar sail ERTBP must have a period which is an integer multiple of 1 year.

In this note we illustrate that a periodic orbit above the ecliptic in the circular case ${ }^{2}$ is not adequate for tracking in the solar sail ERTBP model. For this reason we illustrate two methods for obtaining "better" periodic reference trajectories above the ecliptic for the solar sail ERTBP. The two methods we use are based on (i) a numerical continuation, with the eccentricity $e$ as the perturbation parameter to find a natural orbit in the ERTBP and (ii) a time-delayed feedback mechanism, which can be used when a differential corrector fails to converge.

*, james.biggs@strath.ac.uk, Research Fellow, Department of Mechanical Engineering, University of Strathclyde, Glasgow.

${ }^{\dagger}$ colin.mcinnes@strath.ac.uk, member AIAA, Professor, University of Strathclyde, Glasgow.

¥thomas.waters@nuigalway.ie, Department of Mathematical Physics, National University of Ireland, Galway. 


\section{A. Equations of motion for the elliptic solar sail}

The classical Elliptical Restricted Three-Body Problem (ERTBP) can be modeled conveniently using a pulsating-rotating frame ${ }^{6}$ and in this note we follow the same convention to model the solar sail ERTBP. ${ }^{3}$ We choose our units to set the gravitational constant, the sum of the primary masses, the Earth's semimajor axis about the Sun, and the magnitude of the angular velocity of the rotating frame to be unity. We shall denote by $\mu=3 \times 10^{-6}$ the dimensionless mass of the smaller body $m_{2}$, the Earth, and therefore the mass of the larger body $m_{1}$, the Sun, is given by $1-\mu$. Denoting by $\mathbf{r}_{1}$ and $\mathbf{r}_{2}$ the position of the sail w.r.t. to $m_{1}$ and $m_{2}$, the solar sail's equations of motion in the rotating frame in pulsating-rotating coordinates $x, y, z$ are:

$$
\begin{aligned}
& x^{\prime \prime}-2 y^{\prime}=\frac{1}{1+e \cos f}\left(\frac{\partial \Omega}{\partial x}+a c c_{x}\right) \\
& y^{\prime \prime}+2 x^{\prime}=\frac{1}{1+e \cos f}\left(\frac{\partial \Omega}{\partial y}+a c c_{y}\right) \\
& z^{\prime \prime}+z=\frac{1}{1+e \cos f}\left(\frac{\partial \Omega}{\partial z}+a c c_{z}\right)
\end{aligned}
$$

where $(\cdot)^{\prime}$ denotes differentiation with respect to the true anomaly $f$ and $e=0.0167$ is the eccentricity with

$$
\Omega=\frac{1}{2}\left(x^{2}+y^{2}+z^{2}\right)+\frac{(1-\mu)}{\left\|\mathbf{r}_{1}\right\|}+\frac{\mu}{\left\|\mathbf{r}_{2}\right\|}
$$

and $a c c=\left(a c c_{x}, a c c_{y}, a c c_{z}\right)^{T}$ is the solar sail acceleration defined by:

$$
a c c=\frac{\beta(1-\mu)}{\left\|\mathbf{r}_{1}^{2}\right\|}\left(\hat{\mathbf{r}}_{1} \cdot \hat{\mathbf{n}}\right)^{2} \hat{\mathbf{n}}
$$

where $\beta$ is the solar sail lightness number and is the ratio of the solar sail radiation pressure acceleration to the solar gravitational acceleration, the 'hat' notation denotes the unit vector and $\hat{\mathbf{n}}$ is the unit normal of the sail with respect to the Sun. We define $\hat{\mathbf{n}}$ in terms of two angles $\gamma$ and $\delta$ (radians) in the rotating-pulsating frame:

$$
\hat{\mathbf{n}}=(\cos \gamma \cos \delta, \cos \gamma \sin \delta, \sin \gamma)^{T}
$$

While a $\beta$ value of order $0.3-0.4$ is considered within the realm of possibility, to put the analysis in this paper well within the near-term we will consider very modest $\beta$ values of order 0.05 . Note that the rotatingpulsating coordinates are related to the rotating coordinates $X, Y, Z$ via the equations $X=\rho x, Y=\rho y$ and $Z=\rho z$, with the semi-latus rectum $\rho=\frac{\left(1-e^{2}\right)}{1+e \cos f}$. It follows that when $e=0$ the equations (1) reduce to the circular case with the rotating-pulsating coordinates equal to the rotating coordinates.

\section{Tracking the reference trajectory given in the solar sail CRTBP}

In this section we use a continuous LQR controller in the solar sail ERTBP to track a periodic reference orbit using variations in the sail's orientation. We show that a reference orbit from the solar sail CRTBP is not adequate for tracking in the elliptical case. To measure the quality of the reference orbit given the constraints on the sail, we compute the tracking error. The physical constraints to be considered are the sail's 
maximum deflection (radians) $-\pi / 2 \leq \gamma, \delta \leq \pi / 2$ (the sail has only one reflective side) and its maximum rate of deflection (radians per day) is $-0.42 \leq \dot{\gamma}, \dot{\delta} \leq 0.42$ ( 1 degree per hour). To begin we linearize the equations of motion $\dot{\mathbf{X}}=f(\mathbf{X}, \mathbf{u}(t))$ about the reference orbit $\Gamma$. Writing $\mathbf{x}=\mathbf{X}-\Gamma$ and $\mathbf{u}=\mathbf{u}(t)-\mathbf{u}_{e}$ yields the linear system

$$
\dot{\mathbf{x}}=A(t) \mathbf{x}+B \mathbf{u}
$$

where

$$
A(t)=\partial f(\mathbf{X}, \mathbf{u}(t)) /\left.\partial \mathbf{X}\right|_{\mathbf{X}=\Gamma}, \quad B=\partial f(\mathbf{X}, \mathbf{u}(t)) /\left.\partial \mathbf{u}(t)\right|_{\mathbf{u}(t)=\mathbf{u}_{e}}
$$

From control systems theory the gain matrix $K$ for the linear state feedback control law $\mathbf{u}=-K \mathbf{x}$ which minimizes the quadratic cost function $J=\int_{0}^{\infty} \mathbf{x}^{T} Q \mathbf{x}+\mathbf{u}^{T} R \mathbf{u d t}$ where $Q, R$ are symmetric positive semidefinite weighting matrices and then $K=R^{-1} B^{T} P(t)$ where $P(t)$ is the unique, positive semi-definite solution to the differential Riccati equation:

$$
A(t)^{T} P(t)+P(t) A(t)-P(t) B R^{-1} B^{T} P(t)+Q=\dot{P}(t)
$$

The weights $Q$ and $R$ are free to choose and for simplicity this freedom is reduced to one parameter by letting $Q=I_{6}$ and $R=k I_{2}$, where $k$ is a constant. Therefore, the control effort will be penalized if $k$ is large and the distance from the orbit will be penalized if $k$ is small. The procedure for defining the time-dependent $K=K_{i j}$ matrix is to numerically compute it about the reference orbit and then fit a Fourier function to each component of $K$. The simulation is run for the equivalent of 20 years real time (20 orbits). Although this length of time is longer than the mission time for conventional spacecraft, the solar sail has the ability to perform much longer missions. The results of this are illustrated in Figure 1. When the trajectory begins on

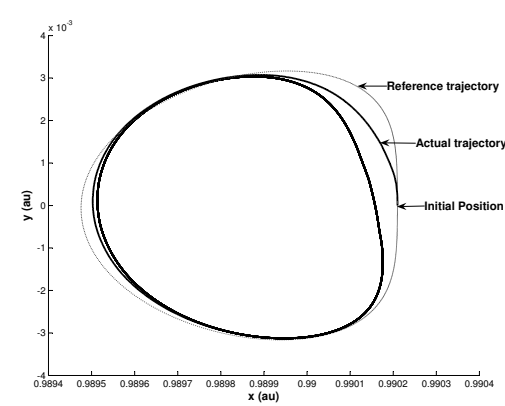

(i)

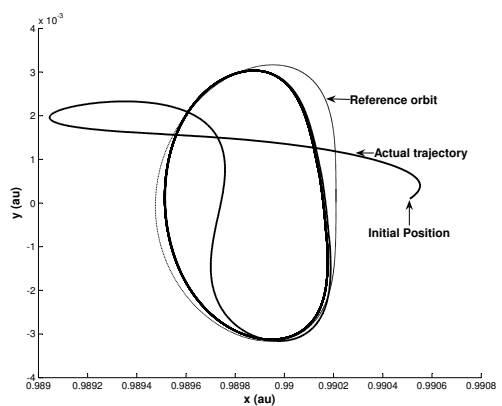

(ii)

Figure 1. LQR control: the thick line is the controlled trajectory and the thin dotted line is the reference orbit (i) initial condition begins on the reference trajectory. (ii) large errors in the initial conditions are included

the reference orbit Figure 1 (i) the solar sail's mean tracking error over 20 years is $1.5556 \times 10^{-4}$ au (23271 $\mathrm{km}$ ). In Figure 1 (ii) we consider initial condition errors of approximately $25000 \mathrm{~km}$ with velocity errors of approximately $180 \mathrm{~m} / \mathrm{s}$. For larger errors the sail does not track the reference orbit. For this case the mean 
tracking error is $2.1985 \times 10^{-4}$ au $(32889 \mathrm{~km})$. These tracking errors are large and in this note we aim to obtain "better" reference trajectories for the elliptic case.

\section{Methods to obtain reference trajectories in the Solar Sail ERTBP}

In this section we propose two methods to obtain suitable periodic reference trajectories above the ecliptic for the solar sail ERTBP. One obvious choice for a reference trajectory would be to use a natural orbit above the ecliptic in the solar sail ERTBP. Therefore, our first method is based on a numerical continuation with the eccentricity, $e$, as the continuation parameter. The second method is based on a time-delayed feedback mechanism which is useful when a trajectories error in periodicity is too large for a differential corrector to close it. We begin here with a description of the numerical continuation.

\section{A. Numerical continuation}

The continuation algorithm is based on a monodromy variant of Newton's method. ${ }^{4}$ The Newton method starts with a trajectory $\mathbf{X}(t)=(x, y, z, \dot{x}, \dot{y}, \dot{z})$ initialized at $t=0$ on a surface of section and provides an iterative improvement to the choice of initial conditions for a periodic orbit:

$$
\mathbf{X}^{*}(0)=\mathbf{X}(0)+(I-M)^{-1}[\mathbf{X}(T)-\mathbf{X}(0)]
$$

where $\mathbf{X}^{*}(0)$ is the improved initial condition and $M$ is the monodromy matrix. We begin with a known periodic orbit at $e=0$ in the solar sail $\mathrm{CRTBP}^{2}$ and continue the parameter $e$ in small increments until the required value $e=0.0167$. The monodromy matrix required for its implementation is derived by recasting the variational equations ((4) with $\mathbf{u}=0$ ) in terms of the state transition matrix (or principle fundamental $6 \times 6$ matrix) $\Phi=\partial \mathrm{X}(t) / \partial \mathrm{X}(0)$, we have $\dot{\Phi}=A(t) \Phi, \quad \Phi(0)=I$. The monodromy matrix $M$ is then defined as $M=\Phi(T)$. Using Newton's method we obtain initial conditions that yield a 1 year periodic orbit above the ecliptic in the Solar Sail ERTBP: $x(0)=0.99026089327975, \quad y(0)=0.00000002531557, \quad z(0)=$ $0.01497820748971, x^{\prime}(0)=0.00000000062302, \quad y^{\prime}(0)=0.00306117310561, \quad z^{\prime}(0)=-0.00000003900302 \quad f(0)=$ $0, \gamma=0.809196, \delta=0$. We note that this periodic orbit is unstable and will require active control to stabilize the solar sail on the orbit. This active control will be implemented using variations in the sail's orientation.

\section{B. Computing reference orbits using a time-delayed feedback mechanism}

In this subsection we propose a novel method for designing reference orbits based on time-delayed feedback control. ${ }^{5}$ This method is particularly useful when the error in periodicity of the initial trajectory is too large for a differential corrector to close the orbit. To illustrate this we use a Monte Carlo simulation of initial conditions (in a small region close to the initial conditions that yield a periodic orbit in the circular case) to find a trajectory as a close as possible to a periodic orbit. This trajectory is illustrated in Figure 2 (i) and we 
note that a differential corrector failed to close this trajectory. To compute a periodic reference trajectory for the nonlinear system $\dot{\mathbf{X}}(t)=f(\mathbf{X}(t), t)$ we use a time-delayed feedback mechanism of the form:

$$
\begin{aligned}
& \dot{\mathrm{X}}(t)=f(\mathbf{X}(t), t)+\mathbf{v}(t) \\
& \mathbf{v}(t)=-K(\mathbf{X}(t)-\mathbf{X}(t-\tau))
\end{aligned}
$$

where $\tau$ is the delay time, which will be $2 \pi$ in order to obtain a 1 year orbit and $K$ is a $6 \times 6$ matrix which is computed experimentally. By inspection of the time-delayed feedback function $\mathbf{v}(t)(7)$ it can be seen that as the trajectory $\mathbf{X}(t)$ approaches periodic i.e. $\|\mathbf{X}(t)-\mathbf{X}(t-\tau)\| \rightarrow 0$, then $\mathbf{v}(t) \rightarrow 0$. The nonlinear equations (7) were simulated until the resulting trajectory satisfied the properties $\|\mathbf{X}(T)-\mathbf{X}(0)\|<\varepsilon$ and $\|\mathbf{v}(t)\|<\partial$ for some pre-specified tolerance parameters $\varepsilon$ and $\partial$.

We note that this method can be implemented using either forward integration or backwards integration and that backward integration worked better in this case. Using the preceding method we obtained the closed reference trajectory in Figure 2 (i). From Figure 2 (ii) it can be seen that the initial orbit requires a large impulse (correction) to close the trajectory but this reduces in magnitude with time. Finally, we
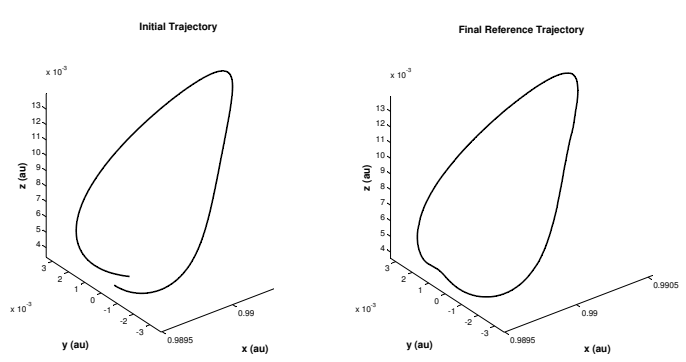

(i)
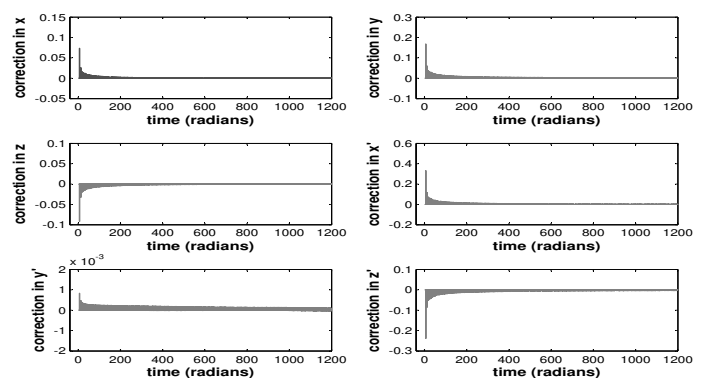

(ii)

Figure 2. Time-delay feedback: (i) this illustrates the initial trajectory and final closed trajectory (ii) the corrections required to close the initial orbit is large but these decrease to small values as time increases.

test the quality of the reference orbits given by the numerical continuation and the time-delayed feedback mechanism in simulation using the LQR method described in Section 2. The results show that there is a vast improvement in the sail's ability to track these reference orbits compared to the reference orbit in the circular case. For the reference orbit computed using the time-delayed feedback method the mean tracking error (without initial condition errors) is $1.522 \times 10^{-5}$ au $(2278 \mathrm{~km})$. Using the same magnitude of errors in initial conditions as the circular case, the mean tracking error is $1.9288 \times 10^{-5}$ au $(2885 \mathrm{~km})$. For the reference orbit found using the numerical continuation the mean tracking error (without initial condition errors) is $4.6733 \times 10^{-8} \mathrm{au}(7 \mathrm{~km})$. This error is small as it is a natural orbit and control is required only to stabilize it. Including the initial condition errors the mean tracking error is $4.2873 \times 10^{-6}$ au $(640 \mathrm{~km})$. 


\section{Conclusions}

This note considers the problem of maintaining a solar sail on a periodic orbit above the ecliptic in the Elliptical Restricted Three-Body Problem (ERTBP). We illustrate that the solar sail does not track a periodic reference orbit obtained from the solar sail Circular Restricted Three-Body Problem adequately. We proceed to illustrate two methods for constructing "better" reference trajectories based on (i) a numerical continuation, with the eccentricity $e$ as the perturbation parameter to find a natural orbit in the ERTBP. (ii) a time-delayed feedback mechanism, which can be used when a differential corrector fails to converge. Both these methods yield reference orbits that significantly improve the tracking error of the solar sail.

\section{Acknowledgments}

This work was funded by grant EP/D003822/1 from the UK Engineering and Physical Sciences Research Council (EPSRC).

\section{References}

${ }^{1}$ McInnes, C. R., 'Solar sailing: technology, dynamics and mission applications'. Springer Praxis, pp. 17-19, 1999.

${ }^{2}$ Waters, T. J., McInnes, C. R., 'Periodic Orbits above the Ecliptic in the Solar sail Restricted Three-body problem'. Journal of Guidance, Control and Dynamics, Vol. 127, pp. 1947-1960, 2007.

${ }^{3}$ Baoyin, H., McInnes, C.R., 'Solar sail equilibria in the elliptical restricted three-body problem'. Journal of Guidance, Control and Dynamics, Vol. 29, No. 3, pp. 538-543, 2006.

${ }^{4}$ Marcinek, R., Pollak, E., 'Numerical methods for locating stable periodic orbits embedded in a largely chaotic system'. Journal of Chemical Physics, 100, 8, pp. 5894-5904, 1994.

${ }^{5}$ Pyragus, K., 'Continuous control of chaos by self-controlling feedback'. Physics Letters A, Vol. 170, pp. 421-428, 1992.

${ }^{6}$ Szebehely, V., 'Theory of Orbits: The restricted problem of three bodies'. Academic Press, New York, pp. 587-595, 1967. 